\title{
ON A CERTAIN CONGRUENCE ASSOCIATED WITH A GIVEN
}

\section{RULED SURFACE*}

BY

\section{E. J. WILCZYNSKI}

In a paper $\dagger$ recently published in these Transactions, I have considered a certain congruence of straight lines, which was intimately associated with a given ruled surface. It is the purpose of the present paper to investigate this congruence more fully, and to complete some theorems which were there stated in a somewhat inadequate form.

Let the system of differential equations

$$
\begin{aligned}
& y^{\prime \prime}+p_{11} y^{\prime}+p_{12} z^{\prime}+q_{11} y+q_{12} z=0, \\
& z^{\prime \prime}+p_{21} y^{\prime}+p_{22} z^{\prime}+q_{21} y+q_{22} z=0,
\end{aligned}
$$

with the independent variable $x$, be given. Let $C_{y}$ and $C_{z}$ be the integral curves of (1), and let $S$ represent its integrating ruled surface, formed by joining corresponding points $P_{y}$ and $P_{z}$ of the two curves.

Let us construct the hyperboloid osculating the surface $S$ along one of its generators $g$. The generators of this hyperboloid, which belong to the same set as $g$, constitute a single infinity of straight lines. There exists such an osculating hyperboloid for every (general) generator of the surface $S$. We obtain, therefore, a congruence $\Gamma$ of straight lines, in which the surface $S$ is contained, and which is uniquely determined by it.

Put

$$
\begin{aligned}
& \rho=2 y^{\prime}+p_{11} y+p_{12} z, \\
& \sigma=2 z^{\prime}+p_{21} y+p_{22} z .
\end{aligned}
$$

Then (Covariants, p. 424), if corresponding points $P_{\rho}$ and $P_{\sigma}$ of the curves $C_{\rho}$ and $C_{\sigma}$ be joined by straight lines, another ruled surface $S^{\prime}$ is obtained

* Presented to the Society (San Francisco) December 20, 1902. Received for publication January 2, 1903.

$\dagger$ Covuriants of systems of linear differential equations and applications to the theory of ruled surfaces, Transactions of the American Mathematical Society, vol. 3, no. 4. I shall refer to it in this paper as Covariants. My other papers will also be quoted by the initial words of their titles. See Covariants, p. 423 , footnote. 
which also belongs to the congruence $\Gamma$. We shall call $S^{\prime}$ the derivative of $S$ with respect to the independent variable $x$.

The system of differential equations, whose integrating ruled surface is $S^{\prime}$ and whose integral curves are $C_{\rho}$ and $C_{\sigma}$, was found to be:

$$
\begin{gathered}
4 J \rho^{\prime \prime}+2\left(J p_{11}+t_{11}\right) \rho^{\prime}+2\left(J p_{12}+t_{12}\right) \sigma^{\prime}+\left(2 J p_{11}^{\prime}-J u_{11}\right. \\
\left.+t_{11} p_{11}+t_{12} p_{21}\right) \rho+\left(2 J p_{12}^{\prime}-J u_{12}+t_{11} p_{12}+t_{12} p_{22}\right) \sigma=0, \\
4 J \sigma^{\prime \prime}+2\left(J p_{21}+t_{21}\right) \rho^{\prime}+2\left(J p_{22}+t_{22}\right) \sigma^{\prime}+\left(2 J p_{21}^{\prime}-J u_{21}\right. \\
\left.+t_{21} p_{11}+t_{22} p_{21}\right) \rho+\left(2 J p_{22}^{\prime}-J u_{22}+t_{21} p_{12}+t_{22} p_{22}\right) \sigma=0,
\end{gathered}
$$

where the notations are the same as in Covariants, p. 438.

If instead of $x$, a new independent variable $\xi$ be introduced by putting

$$
\xi=\xi(x),
$$

and if the quantities which are formed from (1) after the transformation be denoted by a dash, one finds

$$
\begin{aligned}
& \bar{\rho}=\frac{1}{\xi^{\prime}}(\rho+\eta y), \\
& \bar{\sigma}=\frac{1}{\xi^{\prime}}(\sigma+\eta z),
\end{aligned}
$$

The new variable $\xi$ may therefore be chosen in such a way as to make the surface derived from $S$ any ruled surface of the congruence $\Gamma$, excepting $S$ itself together with its osculating hyperboloids.

According to the equations (20) and (56) in Invariants, we find the effect of the transformation of the independent variable upon the quantities $u_{i k}$ to be given by the equations

where

$$
\begin{array}{ll}
\bar{u}_{11}=\frac{u_{11}+2\{\xi, x\}}{\left(\xi^{\prime}\right)^{2}}, & \bar{u}_{12}=\frac{u_{12}}{\left(\xi^{\prime}\right)^{2}}, \\
\bar{u}_{21}=\frac{u_{21}}{\left(\xi^{\prime}\right)^{2}}, & \bar{u}_{22}=\frac{u_{22}+2\{\xi, x\}}{\left(\xi^{\prime}\right)^{2}},
\end{array}
$$

$$
\{\xi, x\}=\frac{\xi^{\prime \prime \prime}}{\xi^{\prime}}-\frac{3}{2}\left(\frac{\xi^{\prime \prime}}{\xi^{\prime}}\right)^{2}=\eta^{\prime}-\frac{1}{2} \eta^{2}
$$

denotes the Schwarzian derivative. Therefore

since

$$
\begin{aligned}
& \bar{I}=\frac{I+4\{\xi, x\}}{\left(\xi^{\prime}\right)^{2}}, \\
& \bar{J}=\frac{J+2\{\xi, x\} I+4\{\xi, x\}^{2}}{\left(\xi^{\prime}\right)^{4}},
\end{aligned}
$$

$$
I=u_{11}+u_{22}, \quad J=u_{11} u_{22}-u_{12} u_{21} \text {. }
$$


Moreover, from the definitions of the quantities $v_{i k}$ and $w_{i k}$ [Invariants, equations (32) and (39)], we find

$$
\begin{aligned}
& \bar{v}_{11}=\frac{1}{\left(\xi^{\prime}\right)^{3}}\left[v_{11}-4 u_{11} \eta+4\{\xi, x\}^{\prime}-8\{\xi, x\} \eta\right], \\
& \bar{v}_{12}=\frac{1}{\left(\xi^{\prime}\right)^{3}}\left[v_{12}-4 u_{12} \eta\right], \\
& \bar{v}_{21}=\frac{1}{\left(\xi^{\prime}\right)^{3}}\left[v_{21}-4 u_{21} \eta\right], \\
& \bar{v}_{22}=\frac{1}{\left(\xi^{\prime}\right)^{3}}\left[v_{22}-4 u_{22} \eta+4\{\xi, x\}^{\prime}-8\{\xi, x\} \eta\right],
\end{aligned}
$$

where

$$
\{\xi, x\}^{\prime}=\frac{d}{d x}\{\xi, x\} \text {, etc. }
$$

Further, we find

$$
\begin{aligned}
\bar{w}_{11}=\frac{1}{\left(\xi^{\prime}\right)^{4}}\left[w_{11}-10 v_{11} \eta+24 u_{11} \eta^{2}-8 u_{11} \eta^{\prime}\right. & +8\{\xi, x\}^{\prime \prime}-40\{\xi, x\}^{\prime} \eta \\
& \left.-16\{\xi, x\}^{2}+40\{\xi, x\} \eta^{2}\right], \\
\bar{w}_{12}= & \frac{1}{\left(\xi^{\prime}\right)^{4}}\left[w_{12}-10 v_{12} \eta+24 u_{12} \eta^{2}-8 u_{12} \eta^{\prime}\right],
\end{aligned}
$$

(9)

$$
\begin{aligned}
\bar{w}_{21}= & \frac{1}{\left(\xi^{\prime}\right)^{4}}\left[w_{21}-10 v_{21} \eta+24 u_{21} \eta^{2}-8 u_{21} \eta^{\prime}\right], \\
\bar{w}_{22}=\frac{1}{\left(\xi^{\prime}\right)^{4}}\left[w_{22}-10 v_{22} \eta+24 u_{22} \eta^{2}-8 u_{22} \eta^{\prime}\right. & +8\{\xi, x\}^{\prime \prime}-40\{\xi, x\}^{\prime} \eta \\
& \left.-16\{\xi, x\}^{2}+40\{\xi, x\} \eta^{2}\right] .
\end{aligned}
$$

Now equations (3) show that, if $J=0$, the derivative of $S$ with respect to $x$ is a developable surface, for then there will be an equation satisfied of the form

$$
\alpha \rho^{\prime}+\beta \sigma^{\prime}+\gamma \rho+\delta \sigma=0 .
$$

This conclusion would not be valid, if in addition to $J=0$, the equations $\alpha=\beta=\gamma=\delta=0$ were also satisfied. In that case we should have, besides $J=0$,

$$
\begin{aligned}
u_{21} v_{12}-u_{22} v_{11} & =0, & u_{21} v_{22}-u_{22} v_{21} & =0, \\
-u_{11} v_{12}+u_{12} v_{11} & =0, & -u_{11} v_{22}+u_{12} v_{21} & =0 .
\end{aligned}
$$


But if $\theta_{4} \neq 0$, we may assume $u_{12}=u_{21}=0$, taking as fundamental curves $C_{y}, C_{z}$ on $S$ the two (distinct) branches of the flecnode curve.* But since

$$
J=u_{11} u_{22}-u_{12} u_{21}=0,
$$

either $u_{11}$ or $u_{22}$ will also vanish. But we have [Covariants, equations (33)]

$$
\begin{aligned}
& 2 \rho^{\prime}+p_{11} \rho+p_{12} \sigma=u_{11} y+u_{12} z, \\
& 2 \sigma^{\prime}+p_{21} \rho+p_{22} \sigma=u_{21} y+u_{22} z,
\end{aligned}
$$

so that in this case also $S^{\prime}$ would be developable.

If however, $\theta_{4}=0$, the flecnode curve has only one branch. Taking it as fundamental curve $C_{y}$ we should find $u_{12}=0$. But from this condition, together with $J=\theta_{4}=0$, would follow

$$
u_{11}=u_{22}=0,
$$

so that according to (10) $S^{\prime}$ would again be developable.

In all cases then, if $J=0$ the derivative of $S$ with respect to $x$ is developable. One can easily see that if $J \neq 0, S^{\prime}$ is not developable, for in that case the planes tangent to $S^{\prime}$ at $P_{\rho}$ and $P_{\sigma}$ intersect the line $L_{y z}$ joining $P_{y}$ and $P_{\text {* }}$ in distinct points, as is shown by equations (10).

We shall therefore obtain all developable surfaces of the congruence $\Gamma$, by finding the most general transformation $\xi=\xi(x)$, which reduces the seminvariant $J$ to zero. For the surface $S$ itself, and its osculating hyperboloids, which alone cannot be obtained as derivatives of $S$, are certainly not developable. Those isolated osculating hyperboloids, which are developable, are also obtained in this way, since for them $J=0$. But according to (7), the most general solution of the differential equation

$$
4\{\xi, x\}^{2}+2 I\{\xi, x\}+J=0,
$$

is the most general independent variable for which $J=0$. To reduce $J$ to zero we may therefore take for $\xi$ the general solution of either of the two equations

$$
\{\xi, x\}=\frac{-I+\sqrt{\theta_{4}}}{4},
$$

$$
\{\xi, x\}=\frac{-I-\sqrt{\theta_{4}}}{4}
$$

where

$$
\theta_{4}=I^{2}-4 J
$$

Although each of the equations $(11 a)$ and $(11 b)$ is of the third order we obtain in this way only two families of $\infty^{1}$ developable surfaces, as we should. For, as equations (4) show, all values of $\xi$ which give the same value to $\eta$ belong

* Since the flecnode curve intersects, in general, every generator of the surface in two points, we speak of it as a curve of two branches. 
to the same developable surface $S^{\prime}$. As a matter of fact, equations $(11 a)$ and (11b) may be written

$$
\eta^{\prime}-\frac{1}{2} \eta^{2}=\frac{-I+\sqrt{\bar{\theta}_{4}}}{4}, \quad \eta^{\prime}-\frac{1}{2} \eta^{2}=\frac{-I-\sqrt{\theta_{4}}}{4},
$$

and these are of the Riccati form, so that the anharmonic ratio of any four solutions is a constant.

We have therefore proved the following theorem:

To every ruled surface $S$ there belongs a congruence $\Gamma$ determined by its osculating hyperboloids. This congruence contains two families of developable surfaces, which coincide if, and only if, $\theta_{4}=0, i$. e., if, and only if, the two branches of the flecnode curve of $S$ coincide. To determine any developable surface of the congruence, it is necessary and sufficient to find a solution of the equation

$$
4\{\xi, x\}^{2}+2 I\{\xi, x\}+J=0,
$$

and to take this solution $\xi=\xi(x)$ as the independent variable of the defining system of differential equations. The derivative of $S$ with respect to $\xi$ will then be a developable surface, and all developable surfaces of the congruence may be obtained in this way. Moreover any four developables of the same family intersect all of the asymptotic tangents of $S$ in point rows of the same cross-ratio.

Let us suppose that the variable $x$ has already been so chosen as to make $J=0$. Then the line $L_{\rho \sigma}$, joining $P_{\rho}$ and $P_{\sigma}$ generates a developable surface of the congruence $\Gamma$, and $C_{\rho}$ and $C_{\sigma}$ are two curves on this surface. Let us assume that $\theta_{4} \neq 0$, and that $C_{y}$ and $C_{z}$ are the two (distinct) branches of the flecnode curve on $S$. Then

$$
u_{12}=u_{21}=0, \quad u_{11}-u_{22} \neq 0, \quad J=u_{11} u_{22}-u_{12} u_{21}=0,
$$

so that either $u_{11}$ or $u_{22}$, but not both, will vanish. Suppose that $u_{11}=0$. Then according to $(10)$

$$
2 \rho^{\prime}+p_{11} \rho+p_{12} \sigma=0,
$$

i. e., if $p_{12} \neq 0, P_{\sigma}$ is a point on the tangent to the curve $C_{\rho}$ described by $P_{\rho}$. In other words $C_{\rho}$ is the cuspidal edge of the developable surface. If $p_{12}$ together with $u_{12}$, were zero, $C_{y}$ would be a straight line, and the curve $C_{\rho}$ would degenerate into a point of this line. The developable surface would be a cone.

If $u_{11} \neq 0, u_{22}$ must vanish, and then $C_{\sigma}$ is the cuspidal edge of the developable surface. This ambiguity corresponds to the fact that every line of the congruence belongs to two of its developable surfaces.

But if $P_{y}$ and $P_{z}$ describe the flecnode curves on $S, P_{\rho}$ and $P_{\sigma}$ are points on the flecnode tangents. Let us call the ruled surface of two sheets, generated 
by the flecnode tangents of $S$, its flecnode surface. Then we have proved the theorem: the focal surfaces of the congruence $\Gamma$ are the two sheets, $F^{\prime \prime}$ and $F^{\prime \prime}$, of the flecnode surface of $S$.

For the focal surfaces of a congruence are the loci of the cuspidal edges of its developable surfaces. The theorem is true also if $\theta_{4}=0$, only in that case $F^{\prime \prime}$ and $F^{\prime \prime}$ coincide.

We can also prove this theorem geometrically. Let $g_{0}, g_{1}, g_{2}, g_{3}$, etc., be consecutive generators of the ruled surface $S$. The hyperboloids $H_{1}, H_{2}$, etc., osculating $S$ along $g_{1}, g_{2}$, etc., are determined respectively by $g_{0}, g_{1}, g_{2} ; g_{1}$, $g_{2}, g_{3}$, etc.' The flecnode tangents $f_{1}^{\prime}, f_{1}^{\prime \prime}$ along generator $g_{1}$ are the two straight lines intersecting $g_{0}, g_{1}, g_{2}, g_{3}$. The flecnode tangents $f_{2}^{\prime}, f_{2}^{\prime \prime}$ of $S$, along $g_{2}$, intersect $g_{1}, g_{2}, g_{3}, g_{4} ; f_{3}^{\prime}, f_{3}^{\prime \prime}$ intersect $g_{2}, g_{3}, g_{4}, g_{5}$; etc. Therefore $g_{3}$ intersects $f_{1}^{\prime}, f_{2}^{\prime}, f_{3}^{\prime}, f_{4}^{\prime}$ as well as $f_{1}^{\prime \prime}, f_{2}^{\prime \prime}, f_{3}^{\prime \prime}, f_{4}^{\prime \prime}$, i. e., four consecutive generators of each sheet of the flecnode surface of $S$. This shows that each of the sheets $F^{\prime \prime}$ and $F^{\prime \prime}$ of the flecnode surface of $S$, has $S$ itself as one of the sheets of its flecnode surface. The congruence $\Gamma$ is made up of the generators of the first kind on the hyperboloids $H_{1}, H_{2}$, etc. $H_{1}$ and $H_{2}$ intersect along the four lines $g_{1}, g_{2}, f_{1}^{\prime} f_{1}^{\prime \prime}$. $\quad H_{2}$ and $H_{3}$ intersect along $g_{2}, g_{3}, f_{2}^{\prime}, f_{2}^{\prime \prime}$, etc. Therefore a generator of the first kind on $H_{1}$ can meet a generator of the first kind on $H_{2}$ only along one of the lines $f_{1}^{\prime}$ or $f_{1}^{\prime \prime}$. Moreover, at every point of $f_{1}^{\prime}$ and $f_{1}^{\prime \prime}$ two such lines actually do meet. It is clear then, that the cuspidal edges of the developable surfaces of the congruence $\Gamma$ must lie on one or the other of the two sheets of the flecnode surface of $S$. This completes the synthetic proof of our theorem.

But we have also seen that each sheet of the flecnode surface of $S$, has $S$ itself as one of the sheets of its flecnode surface.

To prove this analytically as well, we set up the system of differential equations for $F^{\prime}$.

Let us assume $\theta_{4} \neq 0$, and take as fundamental curves $C_{y}$ and $C_{z}$ on $S$ the two branches of the flecnode curve. Then $u_{12}=u_{21}=0$. We may also, by multiplying $y$ and $z$ by properly chosen functions of $x$, make $p_{11}=p_{22}=0$. Under these assumptions eliminate $z, z^{\prime}, \sigma, \sigma^{\prime}$ between equations (1), (2) and (10). If $p_{12} \neq 0$, we find

$$
\begin{array}{r}
y^{\prime \prime}-2 \frac{q_{12}}{p_{12}} y^{\prime}-\rho^{\prime}-q_{11} y+\frac{q_{12}}{p_{12}} \rho=0, \\
\rho^{\prime \prime}+\left(2 q_{22}-\frac{1}{2} p_{12} p_{21}\right) y^{\prime}-2 \frac{q_{12}}{p_{12}} \rho^{\prime}-q_{22} \rho=0 .
\end{array}
$$

A similar system of equations may be obtained for $z$ and $\sigma$. 
Denote by $\mathfrak{U}_{i k}$ the quantities formed from (12) in the same way as are $u_{i k}$ from (1). Then we shall find

$$
\begin{gathered}
\mathfrak{u}_{12}=0, \mathfrak{U}_{21}=4 q_{22}-2 p_{12} q_{21}-8 q_{22} \frac{q_{12}}{p_{12}} \\
\mathfrak{U}_{11}-\mathfrak{U}_{22}=4\left(q_{11}-q_{22}\right)
\end{gathered}
$$

Therefore the curve $C_{y}$ is flecnode curve on $F^{\prime}$ as well as on $S$. If the two branches of the flecnode curve are distinct on $S$, they are also distinct on $F^{\prime \prime}$ and $F^{\prime \prime}$ for $q_{11}-q_{22}$ does not vanish under our assumptions unless $\theta_{4}=0$. To complete the proof that $S$ is a sheet of the flecnode surface of $F^{\prime \prime}$ we have still to show that the flecnode tangents to $F^{\prime}$ constructed, of course, along $C_{y}$ are the generators of $S$. But the flecnode tangent to the surface $F^{\prime \prime}$ at a point $P_{y}$ of the flecnode curve, is the line joining it to the point, whose coördinates are obtained from system (12) in the same way as the coördinates of $P_{\rho}$ are obtained from system (1). But the coördinates of this point are

$$
2 y^{\prime}-\frac{2 q_{12}}{p_{12}} y-\rho=-2 \frac{q_{12}}{p_{12}} y-p_{12} z,
$$

i. e., this point is on the generator of the surface $S$ which passes through $P_{y}$. This completes the proof of our theorem if $p_{12} \neq 0$. But if $p_{12}$, together with $u_{12}$, vanishes, $F^{\prime}$ degenerates into a straight line, and any ruled surface made up of lines intersecting it may be called its flecnode surface. The theorem may therefore be stated as being true in all cases.

The curve $C_{y}$ is one branch of the flecnode curve of $F^{\prime}$. The other may be found by putting (Covariants, $\S 4$ )

$$
Y=\mathfrak{U}_{21} y-\left(\mathfrak{u}_{11}-\mathfrak{U}_{22}\right) \rho .
$$

The flecnode tangents to $F^{\prime}$ along this curve generate the second sheet of the flecnode surface of $F^{\prime}$; but this does not in general belong to the congruence $\Gamma$-never in fact, as we shall see, unless $F^{\prime}$ degenerates into a straight line.

The flecnode surface $F^{\prime}$ may be of the second order. This is so if, and only if,

$$
\mathfrak{u}_{12}=\mathfrak{u}_{21}=\mathfrak{u}_{11}-\mathfrak{U}_{22}=0
$$

But this requires $q_{11}-q_{22}$ to vanish, which condition, together with those already fulfilled, gives

$$
u_{12}=u_{21}=u_{11}-u_{22}=0,
$$

i. e., only if the ruled surface $S$ is of the second order, can a sheet of its flec- 
node surface be of the second order. Its flecnode surface in that case coincides with itself, generated however by the generators of the second set.

Let us put

$$
\begin{aligned}
u_{21} v_{12}-u_{22} v_{11} & =2 J \lambda_{11}, & u_{21} v_{22}-u_{22} v_{21} & =2 J \lambda_{21}, \\
-u_{11} v_{12}+u_{12} v_{11} & =2 J \lambda_{12}, & -u_{11} v_{22}+u_{12} v_{21} & =2 J \lambda_{22} ;
\end{aligned}
$$

whence

$$
\begin{array}{ll}
2\left(u_{11} \lambda_{11}+u_{21} \lambda_{12}\right)=-v_{11}, & 2\left(u_{11} \lambda_{21}+u_{21} \lambda_{22}\right)=-v_{21}, \\
2\left(-u_{12} \lambda_{11}+u_{22} \lambda_{12}\right)=-v_{12}, & 2\left(u_{12} \lambda_{21}+u_{22} \lambda_{22}\right)=-v_{22} .
\end{array}
$$

If we denote the coefficients of (3) by $P_{i k}$ and $Q_{i k}$, so that (3) becomes

we shall have

$$
\begin{aligned}
& \rho^{\prime \prime}+P_{11} \rho^{\prime}+P_{12} \sigma^{\prime}+Q_{11} \rho+Q_{12} \sigma=0, \\
& \sigma^{\prime \prime}+P_{21} \rho^{\prime}+P_{22} \sigma^{\prime}+Q_{21} \rho+Q_{22} \sigma=0,
\end{aligned}
$$

$$
P_{i k}=p_{i k}+\lambda_{i k}
$$

$$
\begin{array}{ll}
Q_{11}=q_{11}+\frac{1}{2}\left(\lambda_{11} p_{11}+\lambda_{12} p_{21}\right), & Q_{21}=q_{21}+\frac{1}{2}\left(\lambda_{21} p_{11}+\lambda_{22} p_{21}\right) \\
Q_{12}=q_{12}+\frac{1}{2}\left(\lambda_{11} p_{12}+\lambda_{12} p_{22}\right), & Q_{22}=q_{22}+\frac{1}{2}\left(\lambda_{21} p_{12}+\lambda_{22} p_{22}\right)
\end{array}
$$

Let us denote by $U_{i k}$ the quantities which are formed from system (15) in the same way as are the quantities $u_{i k}$ from (1). Then we shall find

$$
J U_{12}=J u_{12}+\frac{1}{2}\left(u_{12} w_{11}-u_{11} w_{12}\right)-3 J^{\prime} \lambda_{12},
$$$$
J\left(U_{11}-U_{22}\right)=J\left(u_{11}-u_{22}\right)+\frac{1}{2}\left(u_{21} w_{12}-u_{12} w_{21}+u_{11} w_{22}-u_{22} w_{11}\right)-3 J^{\prime}\left(\lambda_{11}-\lambda_{22}\right) \text {. }
$$

If we assume $u_{12}=u_{21}=0, \theta_{4} \neq 0$, the curves $C_{y}$ and $C_{z}$ are the flecnode curves on $S$, and the curves $C_{\rho}$ and $C_{\sigma}$ on the derived surface $S^{\prime}$ are the intersections of this surface with the flecnode surface of $S$. It may happen that $C_{\rho}$ is one branch of the flecnode curve of $S^{\prime}$. This is so if, and only if, $U_{12}=0$. In other words, the derivative of $S$ has a branch of its flecnode curve on the flecnode surface of $S$, if one of the two conditions

is satisfied.

$$
u_{12}=U_{12}=0 \quad \text { or } \quad u_{21}=U_{21}=0
$$

There exist two families of $\infty^{2}$ non-developable ruled surfaces of the congruence $\Gamma$ each of which has one branch of its flecnode curve on the flecnode surface of $S$. 
This we shall now proceed to prove. Assuming $J \neq 0, U_{12}=0$ will be a consequence of $u_{12}=0$, if

$$
w_{12}-3 v_{12} \frac{J^{\prime}}{J}=0
$$

as is shown by (17). The equation $u_{12}=0$ is left invariant by an arbitrary transformation of the independent variable. If then $C_{y}$ be taken as one branch of the flecnode curve on $S$, the curve $C_{\rho}$ will be a branch of the flecnode curve on $S^{\prime}$ provided the independent variable be so chosen as to satisfy the equation

$$
\bar{w}_{12}-3 \bar{v}_{12} \frac{\bar{J}^{\prime}}{\bar{J}}=0
$$

or, making use of (7), (8) and (9), provided

$$
w_{12}+2 v_{12} \eta-3 v_{12} \frac{J^{\prime}+2\{\xi, x\} I^{\prime}+2\{\xi, x\}^{\prime} I+8\{\xi, x\}\{\xi, x\}^{\prime}}{J+2\{\xi, x\} I+4\{\xi, x\}^{2}}=0,
$$

which equation is of the second order with respect to $\eta$. This proves our assertion, that there exists a family of $\infty^{2}$ ruled surfaces in the congruence $\Gamma$, each such that one of the branches of its flecnode curve lies on $F^{\prime}$. There is another such family connected with the other sheet $F^{\prime \prime}$ of the flecnode surface. The surfaces of the second family are determined by the equation

$(19)^{\prime} \quad w_{21}+2 v_{21} \eta-3 v_{21} \frac{J^{\prime}+2\{\xi, x\} I^{\prime}+2\{\xi, x\}^{\prime} I+8\{\xi, x\}\{\xi, x\}^{\prime}}{J+2\{\xi, x\} I+4\{\xi, x\}^{2}}=0$,

which is obtained from (19) by permuting the indices 1 and 2.

It may happen that the derivative of $S$ has both of the branches of its flecnode curve on the flecnode surface of $S$, one on each sheet. If this is so (assuming $\theta_{4} \neq 0$ ), we shall have simultaneously

whence

$$
u_{12}=u_{21}=U_{12}=U_{21}=0,
$$

$$
w_{12}-3 v_{12} \frac{J^{\prime}}{J}=0, \quad w_{21}-3 v_{21} \frac{J^{\prime}}{J}=0 .
$$

But since $J$ is not zero, this gives

$$
w_{12} v_{21}-w_{21} v_{12}=0
$$

which together with $u_{12}=u_{21}=0$, makes

$$
\Delta=\left|\begin{array}{ccc}
u_{11}-u_{22} & u_{12} & u_{21} \\
v_{11}-v_{22} & v_{12} & v_{21} \\
w_{11}-w_{22} & w_{12} & w_{21}
\end{array}\right|=0 .
$$


But this is the condition under which $S$ belongs to a linear complex (Geom, try, p. 351). The converse is also true, i. e., if $\Delta=0$ and $\theta_{4} \neq 0$, a doub] infinity of surfaces $S^{\prime}$ exists, each of which has the property in question. For equations (19) and (19)' will then be identical, the two (distinct) branches of th flecnode curve on $S$ being taken as fundamental curves.

If however $\theta_{4}$ vanishes, we may still assume $u_{12}=0$, whence follows in thi case $u_{11}-u_{22}=0$. The flecnode surface $F$ of $S$ has only one sheet, and is order that both of the branches of the flecnode curve of $S^{\prime}$ may be on $F$, the: must coincide. We must then in this case choose the independent variable st as to satisfy the simultaneous conditions

$$
u_{12}=U_{12}=u_{11}-u_{22}=U_{11}-U_{22}=0 \text {. }
$$

The first two give, as before

$$
w_{12}-3 v_{12} \frac{J^{\prime}}{J}=0
$$

The last two give

$$
\frac{1}{2}\left[u_{21} w_{12}+u_{11}\left(w_{22}-w_{11}\right)\right]-\frac{3}{2} \frac{J^{\prime}}{J}\left[u_{21} v_{12}+u_{11}\left(v_{22}-v_{11}\right)\right]=0,
$$

whence, substituting the value of $J^{\prime} / J$ from the preceding equation, we find

$$
u_{11}\left[v_{12}\left(w_{22}-w_{11}\right)-w_{12}\left(v_{22}-v_{11}\right)\right]=0 .
$$

Developable surfaces being excluded, $u_{11}$ cannot vanish. Therefore

$$
v_{12}\left(w_{11}-w_{22}\right)-w_{12}\left(v_{11}-v_{22}\right)=0,
$$

which, together with $u_{12}=u_{11}-u_{22}=0$, makes $\Delta$ vanish.

Conversely, if

$$
\Delta=\theta_{4}=0 \text {, }
$$

we can always assume $u_{12}=u_{11}-u_{22}=0$, which gives either $u_{21}=0$, i. e., p surface of the second order, or

$$
v_{12}\left(w_{11}-w_{22}\right)-w_{12}\left(v_{11}-v_{22}\right)=0 \text {. }
$$

If then the independent variable be so chosen as to make

the equations

$$
w_{12}-3 v_{12} \frac{J^{\prime}}{J}=0
$$

$$
U_{12}=U_{11}-U_{22}=0
$$

will follow. The following theorem is therefore true, whether $\theta_{4}$ is zero or not. 
If a non-developable ruled surface of the congruence $\Gamma$ has both of the branches of its flecnode curve on the flecnode surface of $S$, one on each sheet, then the surface $S$ belongs to a linear complex. There exists in this case a double infinity of surfaces of the congruence $\Gamma$, each of which has the property in question. Conversely, if $S$ belongs to a linear complex there exists a double infinity of ruled surfaces in the congruence, whose flecnode curves are thus situated on the flecnode surface of $S$.

In case of a surface of the second order all of these surfaces $S^{\prime}$ as well as its flecnode surface coincide with it, and the theorem, while true, becomes trivial.

If $\Delta$ does not vanish identically, it will, in general, vanish for particular values of $x$. If the flecnodes on a generator of $S$, corresponding to a particular value of $x=a$ for which $\Delta=0$, are distinct, one of two things must take place. Either the osculating hyperboloid hyperosculates the surface along that generator, or else the two flecnodes corresponding to $x=a$ on the derivative are on the flecnode surface of $S$, one on each sheet. For, if we take $u_{12}=u_{21}=0$, the condition that $\Delta$ vanishes gives either

$$
u_{12}=u_{21}=u_{11}-u_{22}=0 \quad \text { or } \quad u_{12}=u_{21}=v_{12} w_{21}-v_{21} w_{12}=0,
$$

for $x=a$.

In the first case the osculating hyperboloid hyperosculates the surface (Geometry, p. 444). In the second case, any solution of (19) will, for $x=a$, also satisfy $(19)^{\prime}$, i. e., for $x=a$ the simultaneous conditions

$$
u_{12}=U_{12}=u_{21}=U_{21}=0
$$

will be satisfied, i. e., $P_{\rho}$ and $P_{\sigma}$, two points on the flecnode surface of $S$, will be on the flecnode curve of $S^{\prime}$. One sees at once how this is to be extended to the case when $\theta_{4}=0$.

Equation (19) can be integrated once. Since we have $u_{12}=u_{21}=0$, we find [Invariants, equations (32) and (39)];

$$
\begin{aligned}
& v_{12}=-p_{12}\left(u_{11}-u_{22}\right), \quad v_{11}-v_{22}=2\left(u_{11}^{\prime}-u_{22}^{\prime}\right), \\
& w_{12}=2 v_{12}^{\prime}+\left(p_{11}-p_{22}\right) v_{12}-p_{12}\left(v_{11}-v_{22}\right) .
\end{aligned}
$$

Divide both members of (19) by $v_{12}$. We find

$2 \frac{v_{12}^{\prime}}{v_{12}}+p_{11}-p_{22}+2 \frac{u_{11}^{\prime}-u_{22}^{\prime}}{u_{11}-u_{22}}+2 \frac{\xi^{\prime \prime}}{\xi^{\prime}}-3 \frac{d \log }{d x}\left[J+2\{\xi, x\} I+4\{\xi, x\}^{2}\right]=0$.

Moreover, since $u_{12}=u_{21}=0$, we have

$$
I=u_{11}+u_{22}, \quad J=u_{11} u_{22} .
$$


Therefore we obtain by integration

$$
\frac{p_{12}^{2}\left(u_{11}-u_{22}\right)^{4}\left(\xi^{\prime}\right)^{2}}{\left(u_{11}+2\{\xi, x\}\right)^{3}\left(u_{22}+2\{\xi, x\}\right)^{3}} e^{\mathcal{\int}\left(p_{11}-p_{22}\right) d x}=c,
$$

where $c$ is a constant. Of course (19)' may be treated in the same manner.

We have seen that there exist $\infty^{2}$ ruled surfaces of the congruence $\Gamma$, the flecnode curve of each of which has one of its branches upon one of the sheets of the flecnode surface of $S$. The question arises, whether among these there exists one (there cannot in general be more than one) whose flecnode surface has one of its sheets in common with that of $S$.

Let us suppose that $F^{\prime}$ is at the same time a sheet of the flecnode surface of $S$ and of $S^{\prime}$. Then $C_{y}$ and $C_{\rho}$ are flecnode curves on $S$ and $S^{\prime \prime}$ respectively, so that we shall have $u_{12}=U_{12}=0$. But more than that, the flecnode tangent to $S^{\prime}$ at any point of $C_{\rho}$ must be a generator of $F^{\prime \prime}$, i. e., must pass through $P_{y}$ and $P_{\rho}$. In other words, the conditions

$$
u_{12}=U_{12}=0, \quad 2 \rho^{\prime}+P_{11} \rho+P_{12} \sigma=\lambda y+\mu \rho
$$

must be simultaneously fulfilled, where $\lambda$ and $\rho$ are some (as yet unknown) functions of $x$. But we have

$$
\begin{gathered}
P_{i k}=p_{i k}+\lambda_{i k}, \\
2 \rho^{\prime}+p_{11} \rho+p_{12} \sigma=u_{11} y+u_{12} z
\end{gathered}
$$

so that our second condition becomes

or

$$
u_{11} y+\lambda_{11} \rho+\lambda_{12} \sigma=\lambda y+\mu \rho,
$$

$$
\left(\lambda_{11}-\mu\right) \rho+\lambda_{12} \sigma=\left(\lambda-u_{11}\right) y \text {. }
$$

But, except for singular values of $x, P_{y}, P_{\rho}$ and $P_{\sigma}$ are not collinear. Therefore we must have

$$
\mu=\lambda_{11}, \quad \lambda=u_{11}, \quad \lambda_{12}=0,
$$

which last equation, together with $u_{12}=0$, gives $v_{12}=0$ if $J \neq 0$. But $u_{12}=v_{12}=0$ is satisfied by either $p_{12}=0$, in which case $F^{\prime}$ degenerates into a straight line, or by

$$
u_{12}=u_{11}-u_{22}=0 \text {. }
$$

But since $U_{12}$ must also vanish, we must have also $w_{12}=0$, which, if $p_{12} \neq 0$, gives the additional condition $u_{21}=0$, i. e., $S$ must be a surface of the second order. 
We have proved the following theorem. If the surface $S$ is not of the second order, and if its flecnode surface has a sheet $F^{\prime}$ which does not degenerate into a straight line, no other ruled surface of the congruence $\Gamma$ has $F^{\prime}$ also as a sheet of its flecnode surface. Or, in other words, the second sheet of the flecnode surface of $F^{\prime}$ does not belong to the congruence $\Gamma$.

If $F^{\prime}$ does degenerate into a straight line, every ruled surface of $\Gamma$ clearly has this straight line as a degenerate sheet of its focal surface.

We have seen that $F^{\prime}$ may degenerate into a straight line. It is, in general, a ruled surface. Can it be a developable surface? If it were developable, according to equations (12) or (10), $p_{12}$ would have to vanish. But the simultaneous conditions $p_{12}=u_{12}=0$ would make $C_{y}$ a straight line. Therefore: if a sheet of a flecnode surface is developable, it degenerates into a straight line. This may also be easily seen geometrically.

Assuming again $u_{12}=u_{21}=0$, we find

$$
\begin{aligned}
& P_{12}=p_{12}+\frac{1}{2 u_{11} u_{22}}\left(-u_{11} v_{12}\right)=\frac{p_{12}}{2 u_{22}}\left(u_{11}+u_{22}\right), \\
& P_{21}=p_{21}+\frac{1}{2 u_{11} u_{22}}\left(-u_{22} v_{21}\right)=\frac{p_{21}}{2 u_{11}}\left(u_{11}+u_{22}\right) .
\end{aligned}
$$

But we can always choose the independent variable so as to make $u_{11}+u_{22}$ vanish. According to (7) it is only necessary, for this purpose, to take as the new independent variable any solution of the equation

which gives the equation

$$
4\{\xi, x\}+I=0,
$$

$$
2\left(2 \eta^{\prime}-\eta^{2}\right)+I=0
$$

for $\eta$. This is of the first order and of the RICCATI form, so that the anharmonic ratio of any four solutions is constant. Moreover the conditions $P_{12}=P_{21}=0$ prove that $C_{\rho}$ and $C_{\sigma}$ are asymptotic lines on $S^{\prime}$. Moreover if $p_{12}$ and $p_{21}$ are not both zero in the above equations, i. e., if $S$ is not contained in a linear congruence it is not only sufficient, but it is necessary to make $u_{11}+u_{22}$ vanish so as to have $P_{12}=P_{21}=0$. We have the following theorem:

If $S$ is a ruled surface with two distinct branches to its flecnode curve and not belonging to a linear congruence, there exists just a single infinity of ruled surfaces in the congruence $\Gamma$, whose intersections with the two sheets of the flecnode surface of $S$ are asymptotic lines upon them. They are the derivatives of $S$ when the independent variable is so chosen as to make the seminvariant I vanish. Moreover, the point-rows in which any four of these surfaces intersect the asymptotic tangents of $S$ all have the same anharmonic ratio. 
In a former paper (Invariants, p. 22) we have adopted a canonical form to which system (1) can always be reduced, namely that, for which

$$
p_{i k}=0, \quad q_{11}+q_{22}=0 .
$$

We can now say that, if a system (1) is written in its canonical form, its integral curves are asymptotic lines on its integrating ruled surface, and its derivative with respect to $x$ is cut by the two sheets of the flecnode surface of $S$ along asymptotic lines.

If $\theta_{4}=0$, since

$$
\theta_{4}=I^{2}-4 J
$$

$J$ also will vanish for the canonical form, so that in this case the surfaces just mentioned will coincide with the (single) family of developable surfaces of the congruence.

If $p_{12}=p_{21}=0$ together with $u_{12}=u_{21}=0$, the flecnode curve of $S$ consists of two straight lines, and every ruled surface of the congruence has the property in question. The reduction to the canonical form must therefore have a different significance in this case. To find it, let us assume that the curves $C_{y}$ and $C_{s}$ are asymptotic lines on $S$, but not at the same time flecnode curves, i. e., let them be any two asymptotic lines different from the straight line directrices. Then $p_{12}$ and $p_{21}$ will vanish, while $u_{12}$ and $u_{21}$ do not. We can, moreover, also assume $p_{11}=p_{22}=0$, so that system (1) has been reduced to the semi-canonical form.

The conditions which are necessary and sufficient to make $S$ belong to a linear congruence are, that all of the minors of the second order in $\Delta$ must vanish (Geometry, p. 356). 'Three of these minors are

$$
\begin{gathered}
\left(u_{11}-u_{22}\right) v_{12}-u_{12}\left(v_{11}-v_{22}\right), \quad\left(u_{11}-u_{22}\right) v_{21}-u_{21}\left(v_{11}-v_{22}\right), \\
u_{12} v_{21}-u_{21} v_{12} .
\end{gathered}
$$

Since we have assumed $p_{i k}=0$,

$$
u_{i k}=-4 q_{i k}, \quad v_{i k}=-8 q_{i k}^{\prime}, \quad w_{i k}=-16 q_{i k}^{\prime \prime} .
$$

Inserting these values in the above minors of $\Delta$, and equating them to zero, we find that the quotients $q_{12} /\left(q_{11}-q_{22}, q_{21} / q_{11}-q_{22}\right)$ must be constants. If they are, the other minors will also vanish. Let us put

$$
q_{12}=a\left(q_{11}-q_{22}\right), \quad q_{21}=b\left(q_{11}-q_{22}\right),
$$

where $a$ and $b$ are constants. Our system (1) now has the form

$$
\begin{aligned}
& y^{\prime \prime}+q_{11} y+a\left(q_{11}-q_{22}\right) z=0 \\
& z^{\prime \prime}+b\left(q_{11}-q_{22}\right) y+q_{22} z=0 .
\end{aligned}
$$


If we compute the coefficients $P_{12}$ and $P_{21}$ of the derived system from (16), we find

$$
P_{12}=\frac{-q_{11} q_{12}^{\prime}+q_{12} q_{11}^{\prime}}{q_{11} q_{22}-q_{12} q_{21}}, \quad P_{21}=\frac{-q_{22} q_{21}^{\prime}+q_{21} q_{22}^{\prime}}{q_{11} q_{22}-q_{12} q_{21}}
$$

But if (24) has the canonical form, we shall have

$$
q_{11}+q_{22}=0
$$

which substituted in (23) gives

$$
q_{22}=-q_{11}, \quad q_{12}=2 a q_{11} \quad q_{21}=2 b q_{11},
$$

whence

$$
P_{12}=P_{21}=0
$$

i. e., $C_{\rho}$ and $C_{\sigma}$ are asymptotic lines on $S^{\prime}$. We have therefore the following additional theorem :

If a ruled surface $S$ is contained in a linear congruence, there exists a single infinity of ruled surfaces $S^{\prime}$ in this congruence, whose intersections with the developable surfaces made up of the tangents to the asymptotic curves of $S$, are asymptotic lines upon them. The point-rows, in which any four of these surfaces $S^{\prime}$ intersect the tangents to the asymptotic lines of $S$, all have the same cross-ratio. Any one of these surfaces is obtained by taking the derivative of $S$ with respect to an independent variable which is so chosen as to make the seminvariant I vanish.

We might say more briefly that, in this case the asymptotic lines of $S$ and its derivative correspond to each other.

In a former paper (Covariants, p. 439) we have investigated a special case of this relation between $S$ and $S^{\prime}$, namely that in which systems (1) and (15) assume the semicanonical form simultaneously. This gives rise to the further condition that all of the coefficients in (24) must be constants. We saw then, that there are three distinct types of surfaces for which this takes place. Their equations may be written respectively

$$
\begin{gathered}
x_{1}^{\mu} x_{4}^{\lambda}-x_{2}^{\mu} x_{3}^{\lambda}=0, \\
x_{3}-x_{4} e^{\lambda x_{1} / x_{2}}=0, \\
x_{3} x_{4}^{2}-3 x_{1} x_{2} x_{4}+2 x_{1}^{3}=0,
\end{gathered}
$$

where $\lambda$ and $\mu$ are any constants. The last equation represents CAYLEY's 
cubic scroll. The proof of this theorem there given is not quite adequate, owing to the fact that in the discussion of the last two cases a form of the equations was adopted for which $J=0$. But it is easy to complete the demonstration.

Univ kritity of California,

Berkeley, December 15, 1902. 